\title{
Ordenamiento
}

\section{ecológico territorial de Guadalupe Cuautepec, San Juan Bautista Suchitepec, Oaxaca, desde una perspectiva técnica y comunitaria}

\section{Ecological Zoning of Guadalupe Cuautepec, San Juan Bautista Suchitepec, Oaxaca, from a technical and community perspective}

\author{
Gabriela Álvarez-Olguín*, Fidencio Sustaita-Riveral', Gilberto Bautista-Sánchez’ y Eucebio César Pedro-Santos'
}

1 Instituto de Hidrología, Universidad Tecnológica de la * Autor de correspondencia. galvarez@mixteco.utm.mx Mixteca.

\section{RESUMEN}

En la comunidad de Guadalupe Cuautepec, la deforestación, el sobrepastoreo y las prácticas agrícolas insostenibles, han provocado la erosión del suelo y en consecuencia el azolvamiento de la laguna ubicada en la localidad. Con el fin generar información básica para la creación de estrategias de protección de este cuerpo de agua, se elaboró un ordenamiento ecológico territorial a partir del diagnóstico de los recursos agua, suelo y vegetación, de la comunidad. Desde el punto de vista técnico se realizó una caracterización socioeconómica y biofísica; esta última incluyó aspectos sobre: geomorfología, hidrología, geología, edafología, vegetación, erosión, uso potencial del suelo, conflictos de uso e inestabilidad de suelos. Por otro lado, se llevó a cabo un diagnóstico rural participativo, en el que se identificaron los problemas más relevantes relacionados con los recursos naturales y las acciones que la comunidad realizará para el manejo sustentable de los mismos. Se establecieron objetivos de alta prioridad para lograr el manejo sustentable de los recursos naturales, entre los que están: realizar obras para la conservación de suelos, controlar el aprovechamiento forestal y reforestar zonas sin vegetación. Se estimó que $77.8 \%$ del territorio de Cuautepec, presenta alto y muy alto riesgo de erosión, con índices superiores a 50 t/ha/año. Se determinó que los terrenos más erosionados con necesidades de restauración abarcan 609.34 ha y que la superficie prioritaria a reforestar es de 439.52 ha.

Palabras Clave: conflictos de uso, diagnóstico rural participativo, erosión del suelo, región Mixteca, uso potencial del suelo.

\section{ABSTRACT}

In the Guadalupe Cuautepec community, deforestation, overgrazing and unsustainable agricultural practices, have caused soil erosion and hence the siltation of the lagoon located in the town. In order to generate basic information to create protective strategies to this water body, an ecological zoning was developed from the diagnosis of this community resources': water, soil and vegetation. From the technical point of view a socioeconomic and biophysical characterization was performed; the latter included geomorphological, hydrologic, geological, soil, vegetation, erosion, potential land use, land use conflicts and ground instability. On the other hand, a participatory rural appraisal was conducted, in which the most relevant problems related to natural resources and the community actions needed for the sustainable management of such resources were identified. Top priority targets were set to achieve sustainable management of natural resources, among which are: soil conservation works, controlled logging activities and reforestation of non-vegetated areas. It was estimated that $77.8 \%$ of the Cuautepec territory presents high and very high risk of erosion, with indices above than 50 t/ha/ year. It was determined that the most eroded lands in need of restoration cover 609.34 ha, and that the priority area to reforest is 439.52 ha.

KEYWORDS: land use conflicts, participatory rural appraisal, soil erosion, Mixteca region, potential land use. 


\section{INTRODUCCIÓN}

Los recursos naturales son elementos indispensables para la existencia humana, sin embargo, la presión que se ejerce sobre estos tiene efectos negativos sobre el ambiente. En México, los problemas de degradación de suelos, deforestación, sobreexplotación de recursos hídricos y pérdida de biodiversidad, dejaron de considerarse como simples datos estadísticos para constituir la causa de numerosos conflictos sociales (Cloter, 2004). El país conserva solo aproximadamente $50 \%$ de su cobertura de vegetación natural y se han perdido 127 especies vegetales y animales de las que 74 (58.2\%) eran endémicas (Conabio, 2009).

En la región Mixteca, los índices de deforestación y degradación del suelo son muy elevados, motivados por una agricultura insostenible y el sobrepastoreo (Blanco et al., 2001). El constante cambio de uso de suelo ha generado en forma paulatina la pérdida de la cobertura vegetal, la cual protege al suelo de la erosión, favorece los procesos de infiltración y la recarga de los acuíferos. La comunidad de Guadalupe Cuautepec del municipio de San Juan Bautista Suchitepec, Oaxaca, no está aislada de esta problemática; no obstante sus habitantes han realizado diversas actividades para la restauración y conservación de sus recursos naturales, motivados por la necesidad de proteger, contra el azolvamiento, a la laguna que se encuentra en esta localidad. En 1998 iniciaron las actividades, con la exclusión del pastoreo de un área de 100 ha, y en 2007 se construyeron dos obras para retener sedimentos en corrientes que desembocan en la laguna. Si bien, estas acciones han ayudado a disminuir la entrada de sedimentos al cuerpo de agua, podrían ser ineficientes dado que no tienen un respaldo técnico que las justifique. Por lo anterior, la solución de los problemas de degradación se debe basar en el conocimiento del estado actual de los recursos naturales en la comunidad, no obstante, en el diagnóstico de los mismos un factor clave es la participación de los miembros de la comunidad. En el diagnóstico participativo se identifican los problemas que la comunidad considera más importantes, a través de la interacción de la comunidad no solo diagnóstico, sino también en el seguimiento y evaluación del proceso participativo (Expó- sito, 2003; Osorio y Contreras, 2009). El ordenamiento ecológico territorial podrá ser utilizado por los habitantes de Cuautepec como base para la planeación de actividades de protección de la laguna contra el azolvamiento, así como para la restauración y conservación de los recursos naturales.

\section{OBjetivo}

Elaborar el ordenamiento ecológico territorial de Guadalupe Cuautepec, con base en el diagnóstico biofísico y socioeconómico de la localidad, desde una perspectiva comunitaria y utilizando técnicas de percepción remota.

\section{MATERIALES Y MÉTODOS}

Guadalupe Cuautepec es una localidad que pertenece al municipio de San Juan Bautista Suchitepec, Oax.; tiene un territorio de 2493.9 ha, comprendido entre las coordenadas geográficas $97^{\circ} 41^{\prime} 33.5^{\prime \prime}$ y $97^{\circ} 37^{\prime} 17.9$ "' longitud Oeste, y $18^{\circ}$ 3'56.4” y $17^{\circ} 59^{\prime} 47.3^{\prime \prime}$ latitud Norte. Limita al norte con Santiago Chazumba, al noreste con Santa Catarina Zapoquila, al noroeste con San Pedro y San Pablo Tequixtepec y al suroeste con Santiago Miltepec (Fig. 1). Esta zona corresponde a la región Mixteca, que se caracteriza por un relieve intrincado y de diversa fisonomía, tanto por altitud como por el estilo morfoestructural; además, pertenece a la subprovincia fisiográfica de montañas y valles del Occidente de Oaxaca (García-Mendoza, 2004).

La caracterización socioeconómica se llevó a cabo a partir de datos de Inegi (2010). Los aspectos considerados fueron: población, educación, vivienda y servicios básicos, salud, uso del agua, organización local, infraestructura vial y de transporte, actividades productivas y fuentes de empleo.

La caracterización biofísica del territorio realizada a través de técnicas de percepción remota, consistió en determinar los aspectos sobre: geomorfología, hidrología, geología, edafología, vegetación, erosión, uso potencial y conflictos de uso del suelo. Para caracterizar los tipos de vegetación y suelo, se definieron 3 transectos en dirección Este a Oeste considerando la poligonal que define los 


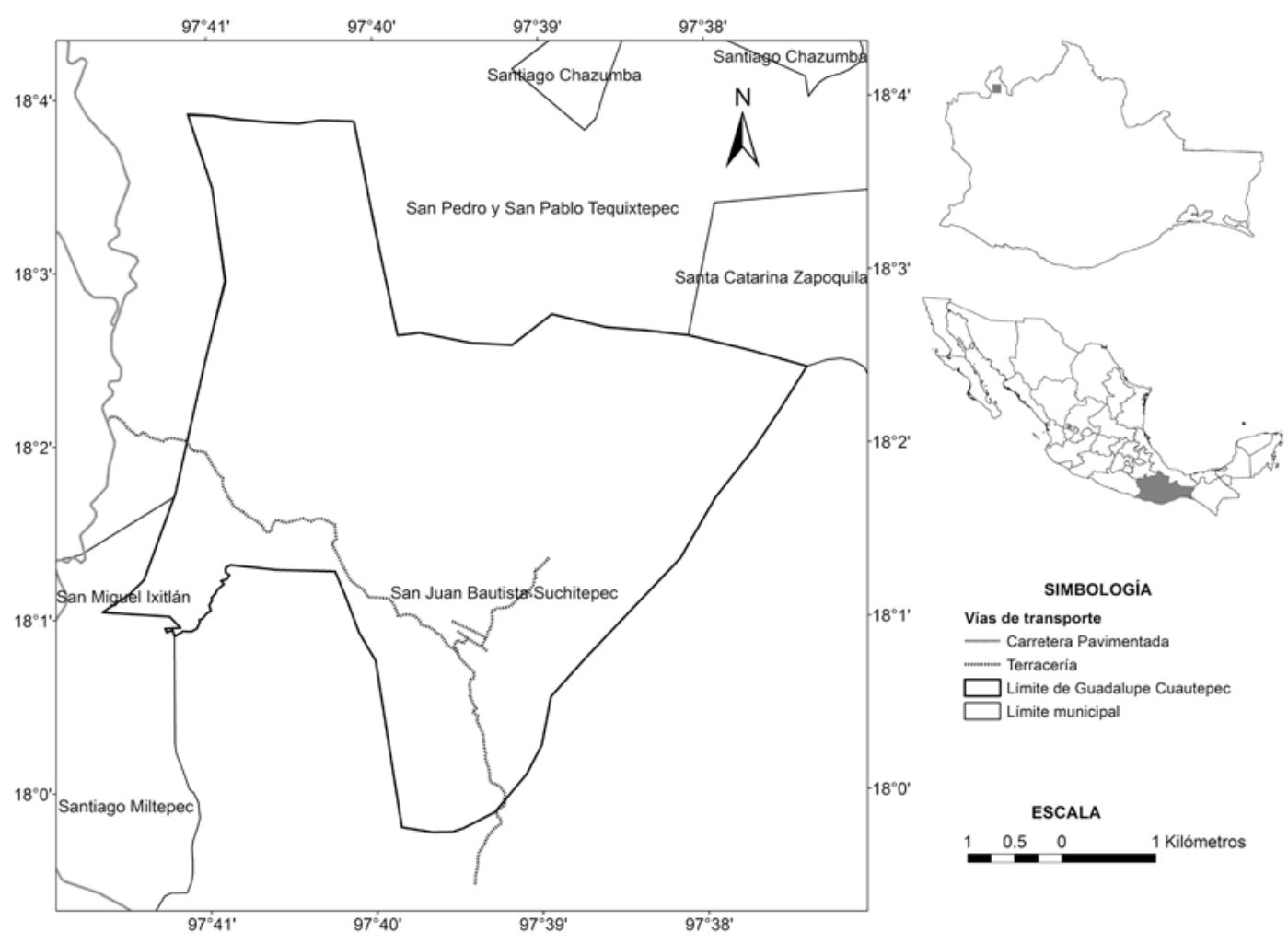

Figura 1. Localización del área de estudio.

terrenos de la comunidad y empleando ortofotos digitales escala 1:20 000, sobre las cuales se ubicaron 12 sitios de muestreo.

Las magnitudes físicas (curva hipsométrica, pendiente del terreno y de los cauces principales) de tres microcuencas que inciden en el territorio de Guadalupe Cuautepec, se obtuvieron a partir de un modelo de elevación digital de Inegi (2003) con resolución de 30 m, manipulado con el programa ArcGis versión 9.1. El parteaguas y la red de drenaje de las microcuencas, se trazaron sobre el producto digital "SРОTView" del área estudiada, el cual corresponde a una imagen de satélite del SPOT 5 y procesada con el formato DIMAP. La fecha de toma de la imagen es del 20 de enero de 2010, con identificación K592/J314, a color y $2.5 \mathrm{~m}$ de resolución.

La erosión del suelo, se determinó empleando la Ecuación Universal de Pérdida de Suelo Modificada (EUPS), que considera la pérdida potencial del suelo por erosión hídrica (Ecuación 1).
$A=R K L S C P$

Donde:

$A=\quad$ promedio anual de pérdida de suelo $\left(\mathrm{t} \mathrm{ha}^{-1}\right)$.

$R=$ factor de erosividad de la lluvia $\left(\mathrm{MJ} \mathrm{mm} \mathrm{ha}^{-1} \mathrm{~h}^{-1}\right)$.

$K=$ factor de erosionabilidad del suelo $\left(\mathrm{t}\right.$ ha $\mathrm{h} \mathrm{MJ}^{-1}$ $\left.\mathrm{mm}^{-1} \mathrm{ha}^{-1}\right)$.

$L S=$ factor de longitud y grado de pendiente (adimensional).

$C=$ factor de manejo de cultivos (adimensional).

$P=\quad$ factor de prácticas mecánicas de control de erosión (adimensional).

El índice de erosividad de la lluvia se calculó con la ecuación (2) en la que la intensidad de la lluvia se cuantificó a partir de datos pluviales registrados en la estación Cristo Rey, ubicada en Cuautepec, a cargo de la Universidad Tecnológica de la Mixteca. 
$R=\sum_{j=1}^{n}\left(E I_{30}\right)_{i}$

$E I_{30}=0.11897+0.0873 \log I I 30$

Donde:

$E I_{30}=$ índice de erosividad para un evento $i$.

$E=\quad$ energía cinética total de la lluvia $\left(\mathrm{MJ} \mathrm{ha}^{-1}\right)$.

$I_{30}=$ intensidad máxima de la lluvia en 30 minutos $\left(\mathrm{mm} \mathrm{h}^{-1}\right)$.

$n=\quad$ número de eventos durante el año.

El factor erosionabilidad del suelo $(K)$ depende de la textura, estructura, contenido de humedad y contenido de materia orgánica de los suelos. El factor $K$ se obtuvo a partir de la unidad de suelo y su textura de los suelos del área de estudio, según la metodología de FAO (1980).

El factor longitud y grado de pendiente $(L S)$ se obtuvo con el álgebra de mapas de ArcGIS versión 9.1, a partir del modelo de elevación digital (MED). El mapa de pendiente (S) se calculó con el módulo "slope" y se aplicó la ecuación (4) en la que el valor $\lambda$ se fijó en 30 , que es la resolución del $M E D$; la constante $m$ fue de 0.5 .

$L S=(\lambda / 22.1)^{m}\left(0.65+0.145 S+0.0065 S^{2}\right)$

Finalmente, el factor $C$ se obtuvo a partir de información propia generada en recorridos detallados de campo, pues la información digital de Inegi no fue de utilidad para este estudio, debido a la escala de trabajo con la cual se generó el mapa de uso actual del suelo. El factor $P$ se consideró igual a 1 , ante la falta de obras de conservación de suelos en la mayor parte del territorio.

Los conflictos de uso de la tierra surgen cuando el uso actual de la tierra es diferente de su capacidad de uso (uso potencial), entendida como el potencial del suelo para soportar actividades agropecuarias y forestales a largo plazo y que si se realizan adecuadamente no afectan la capacidad productiva del suelo. El conflicto de uso permite comparar el uso actual y el uso potencial del suelo, para identificar áreas que pueden degradarse como consecuencia de usos inadecuados, así como para planificar actividades de manejo de los recursos naturales. Este conflicto se origina por sobreuso debido a actividades agrícolas o ganaderas en tierras de capacidad forestal; así como por uso agrícola, ganadero o plantaciones forestales en tierras de capacidad de protección, entre otras.

Para la determinación del uso potencial del suelo se empleó la clasificación conocida como capacidad agrológica, propuesta por el Servicio de Conservación de Suelos del Departamento de Agricultura de los Estados Unidos de América (Klingebiel y Montgomery, 1961).

La clasificación agrológica consiste en caracterizar el territorio, basado en las limitantes que presente el suelo y el grado o magnitud estimada de la limitante, de tal manera que se generan ocho clases donde las limitaciones del suelo en cuanto al uso aumentan progresivamente desde clase I a la VIII en términos del uso agrícola más intensivo posible. Los suelos en una unidad de capacidad son lo suficientemente uniformes para producir clases similares de cultivos, con tratamientos similares de manejo, requerimientos similares conservacionistas y productividad potencial comparable.

Las propiedades de los suelos que afectan el uso potencial se infirieron de la morfología y características asociadas (pendiente, erosión actual, pedregosidad o rocosidad, riesgo de inundación) que generan grupos de unidades de capacidad con los mismos problemas principales de conservación, tales como: erosión, escurrimiento, exceso de agua, limitaciones en zona radicular o limitaciones climáticas.

Se elaboró una matriz de asignación de usos mediante el empleo de ArcGIS 9.1, que consistió en cruzar las categorías del mapa de uso potencial con las categorías de mapa de uso actual y así identificar las áreas con conflictos en el uso del suelo. Después de identificar las áreas con sobreuso del suelo se buscó convertirlas a usos correctos mediante las actividades planeadas en el plan de ordenamiento ecológico del territorio.

Desde el punto de vista comunitario, se realizó un diagnóstico de los recursos naturales a través de cuatro talleres en los que participaron 36 miembros de la comunidad: 13 mujeres y 23 hombres. Las técnicas y actividades 
realizadas fueron: a) cartografía social, en la que se elaboraron mapas de localización de la comunidad sobre la situación de los recursos agua, suelo, vegetación y fauna; b) árbol de problemas, obtenido a partir de la técnica de lluvia de ideas para determinar la causa y efecto de la degradación de los recursos naturales; y c) árbol de objetivos (soluciones), en el que se consideraron los medios y fines a partir de las causas y efectos detectados en el árbol de problemas. Con estos elementos los participantes dialogaron y analizaron el problema del manejo de los recursos naturales e hicieron sus aportaciones desde su percepción y situacionalidad, para las alternativas de solución; partiendo de sus limitaciones y de sus potencialidades (Bautista-Sánchez et al., 2013).

\section{RESULTADOS}

\section{Aspectos socioeconómicos:}

En el 2010, la población fue 113 habitantes, el grupo de edad con más integrantes fue el de más de 60 años con 35 integrantes, lo cual indica que la población está envejeciendo debido a la migración, mientras que la población joven está disminuyendo. La escolaridad promedio es de cuarto grado y el nivel de analfabetismo es de $18.6 \%$. Los habitantes se rigen por usos y costumbres.

La cobertura de energía eléctrica es de $98 \%$, sin embargo la de agua y drenaje sanitario son bajas, ya que solo $27 \%$ de las viviendas particulares cuentan con agua entubada dentro del terreno y con fosas sépticas (Tabla 1). Por otro lado, la comunidad no cuenta con relleno sanitario, por lo que sus residuos sólidos no biodegradables son quemados. Con respecto al transporte, solo existen caminos de terracería y no se tiene servicio público. Existe una clínica donde se atienden enfermedades menores y solo $30 \%$ de los habitantes no tienen derecho a recibir servicios médicos en alguna institución pública.

Las actividades productivas de la comunidad son la agricultura de temporal y la ganadería, aunque existen parcelas menores a una hectárea, donde se aplican riegos de auxilio. Los principales cultivos son maíz y frijol bajo el sistema milpa (asociación de cultivos maíz/frijol/cala-
TAbla 1. Principales características socioeconómicas de la comunidad de Guadalupe Cuautepec.

\begin{tabular}{|c|c|}
\hline \multicolumn{2}{|l|}{ Población } \\
\hline Grupos de edades & $\begin{array}{l}\text { No. de } \\
\text { habitantes }\end{array}$ \\
\hline O a 14 años & 20 \\
\hline 15 a 24 años & 12 \\
\hline 25 a 49 años & 13 \\
\hline 50 a 60 años & 33 \\
\hline 60 y más años & 35 \\
\hline \multicolumn{2}{|l|}{ Características educativas } \\
\hline Grupos de edades & $\begin{array}{l}\text { No. de } \\
\text { habitantes }\end{array}$ \\
\hline 3 a 14 años que no asiste a la escuela & 2 \\
\hline 15 a 24 años que asiste a la escuela & 1 \\
\hline 15 años y más analfabeta & 21 \\
\hline \multicolumn{2}{|l|}{ Servicios de salud } \\
\hline Condición & $\begin{array}{l}\text { No. de } \\
\text { habitantes }\end{array}$ \\
\hline Sin derechohabiencia a servicios de salud & 34 \\
\hline Derechohabiente del IMSS & 67 \\
\hline Derechohabiente del ISSSTE & 5 \\
\hline Derechohabiente del ISSSTE estatal & 0 \\
\hline $\begin{array}{l}\text { Derechohabiente del seguro popular o Seguro } \\
\text { Médico para una Nueva Generación }\end{array}$ & 12 \\
\hline \multicolumn{2}{|l|}{ Servicios públicos básicos } \\
\hline Tipo de servicio & $\begin{array}{c}\text { Cobertura } \\
\text { (\%) }\end{array}$ \\
\hline Energía eléctrica & 98 \\
\hline Agua entubada dentro del terreno de la vivienda & 27 \\
\hline Drenaje conectado a fosa séptica & 27 \\
\hline Sanitario sin admisión de agua & 89 \\
\hline
\end{tabular}

IMSS: Instituto Mexicano del Seguro Social.

ISSSTE: Instituto de Seguridad y Servicios Sociales de los Trabajadores del Estado. 
baza). En el caso de la ganadería, se cría ganado vacuno, que es de tipo extensivo, en menor proporción a la cría de ganado ovino, caprino, equino y asnal, así como animales de traspatio. Otra actividad es la acuicultura, la cual se realiza en estanques y en la laguna. Algunas personas se dedican al tejido de la palma.

\section{Características biofísicas:}

La mayor parte del territorio de Guadalupe Cuautepec (87\%), está comprendido dentro de tres microcuencas cuyas corrientes principales se denominan respectivamente: cañada San Francisco, cañada Grande y barranca Puente de vigas (Fig. 2). Las microcuencas Sur (cañada San Francisco) y Centro (cañada Grande), forman parte de la subcuenca del río Mixteco, mientras que la microcuenca Norte (barranca Puente de vigas) pertenece a la subcuenca del río Acatlán. Ambas subcuencas están dentro de la cuenca del río Atoyac, de la región hidrológica No. 18 (río Balsas). Las características geomorfológicas obtenidas, se describen en la tabla 2. La superficie de las microcuencas es de 3266.9 ha, de la cual, $87.6 \%$ corresponde a Cuautepec.

Con respecto a la vegetación y uso del suelo, la unidad de origen antropogénico que abarca mayor superficie es el uso pecuario con 566.3 ha $(22.7 \%$ de la superficie), mientras que las unidades naturales más extensas en función de la especie dominante (clasificación propia), corresponden a selva baja caducifolia, enebro-tepehuaje y encino-enebro que en conjunto abarcan $60.5 \%$ de la superficie (Fig. 3). Los usos agrícola y urbano, solo abarcan $1.4 \%$ y $0.9 \%$ de la superficie, respectivamente. Además existe una zona inestable por deslizamientos de tierra que ocupa $1.5 \%$ del total.

La mayor parte del territorio $(77.8 \%)$ presenta alto y muy alto riesgo de erosión, con índices superiores a $50 \mathrm{t} /$ ha/año (Fig. 4). Este riesgo se localiza principalmente en las zonas altas y de pendiente fuerte, así como en las áreas con escasa cobertura vegetal, pie de monte e inclusive en partes bajas. Solamente $3.6 \%$ de la superficie presenta erosión ligera y corresponde a los terrenos agrícolas ubicados en las partes planas y a zonas con cobertura vegetal.
Tabla 2. Parámetros geomorfológicos de las microcuencas delimitadas para la comunidad de Guadalupe Cuautepec.

\begin{tabular}{cccc}
\hline \multirow{2}{*}{ Descripción } & \multicolumn{3}{c}{ Microcuencas } \\
\cline { 2 - 4 } & Norte & Centro & Sur \\
\hline Área de la cuenca (ha) & 1320.8 & 817.7 & 1128.4 \\
Perímetro del parteaguas (km) & 17.38 & 13.98 & 16.91 \\
Longitud de la cuenca (km) & 4.70 & 4.80 & 5.40 \\
Índice de forma & 0.60 & 0.35 & 0.39 \\
Coeficiente de compacidad & 1.35 & 1.38 & 1.42 \\
Relación de elongación & 0.87 & 0.67 & 0.70 \\
Altitud media (m) & 2091.16 & 2121.86 & 2173.77 \\
Pendiente media de la cuenca (\%) & 30.30 & 31.30 & 39.70 \\
Orden de corriente & 4.00 & 3.00 & 3.00 \\
Longitud del cauce principal (km) & 5.20 & 5.60 & 6.40 \\
Pendiente del cauce principal (\%) & 4.77 & 3.86 & 4.81 \\
Tiempo de concentración (min) & 0.22 & 0.26 & 0.26 \\
Densidad de drenaje km/km ${ }^{2}$ & 2.79 & 3.14 & 2.38 \\
Frecuencia de corrientes $\mathrm{km}^{-2}$ & 3.79 & 5.14 & 3.99 \\
\hline
\end{tabular}

Por otro lado, $18.6 \%$ de los terrenos presentan erosión moderada, los cuales se localizan en zonas de pendiente entre $10 \%$ y $40 \%$, además de áreas con cobertura vegetal mayor a $20 \%$ y menor a $75 \%$.

Las zonas aptas para la agricultura representan $3.2 \%$ del territorio, las que tienen uso potencial mixto (agrícolaganadería), 23.8\%; los suelos aptos como bosques y restauración abarcan $29.6 \%$ y, los de conservación y protección representan $43.4 \%$ (Fig. 5). Se identificó que $23.9 \%$ del área de la comunidad se encuentra en conflicto de uso, el cual consiste principalmente en la pérdida de áreas forestales o agrícolas debido a la actividad ganadera, mientras que $75.6 \%$ de los terrenos no presentan conflicto (Fig. 6).

\section{Diagnóstico participativo, plan de} acción comunitario y ordenamiento territorial:

Como describieron Bautista-Sánchez et al. (2013), el diagnóstico participativo permitió identificar desde el punto 


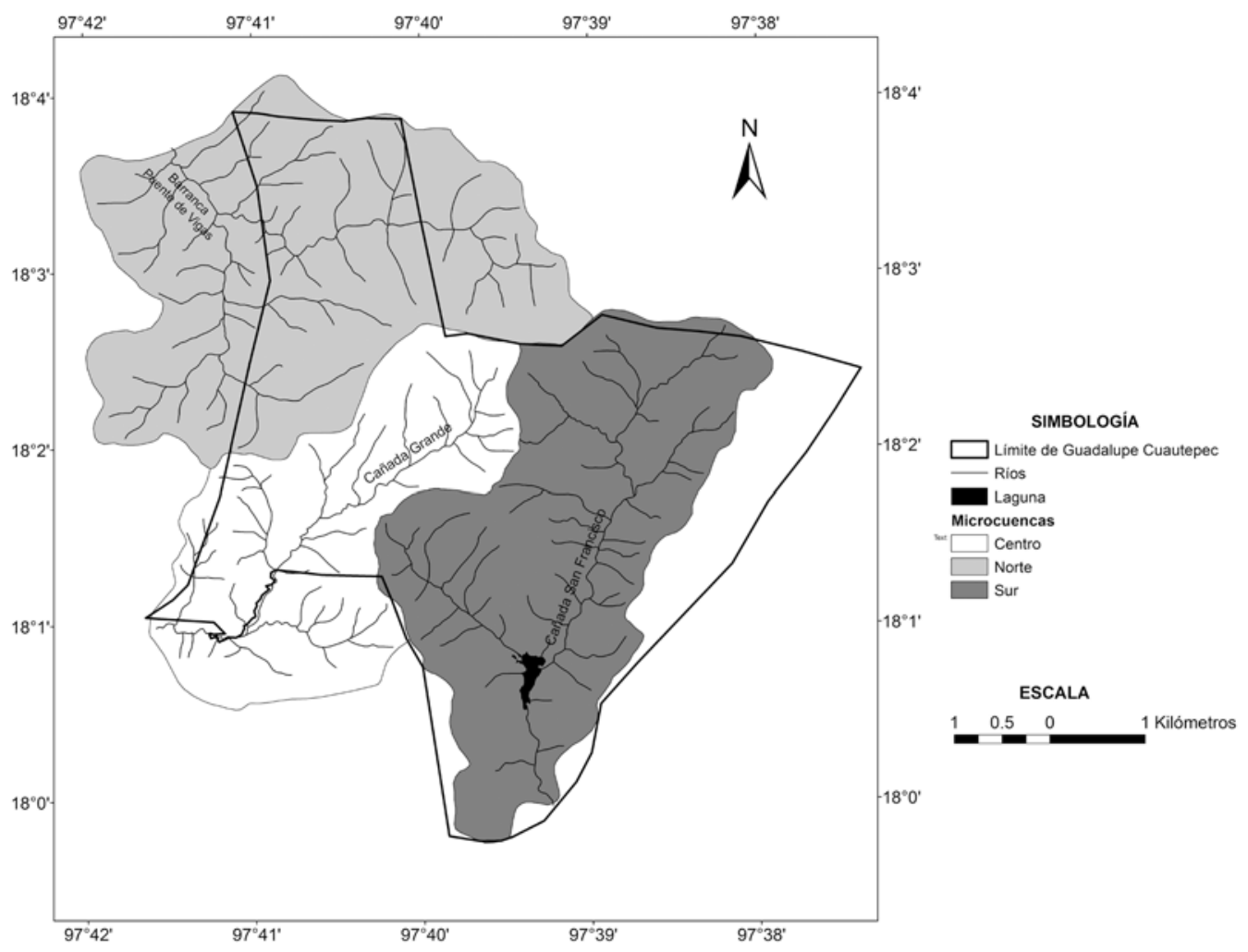

FIGURA 2. Localización de microcuencas.

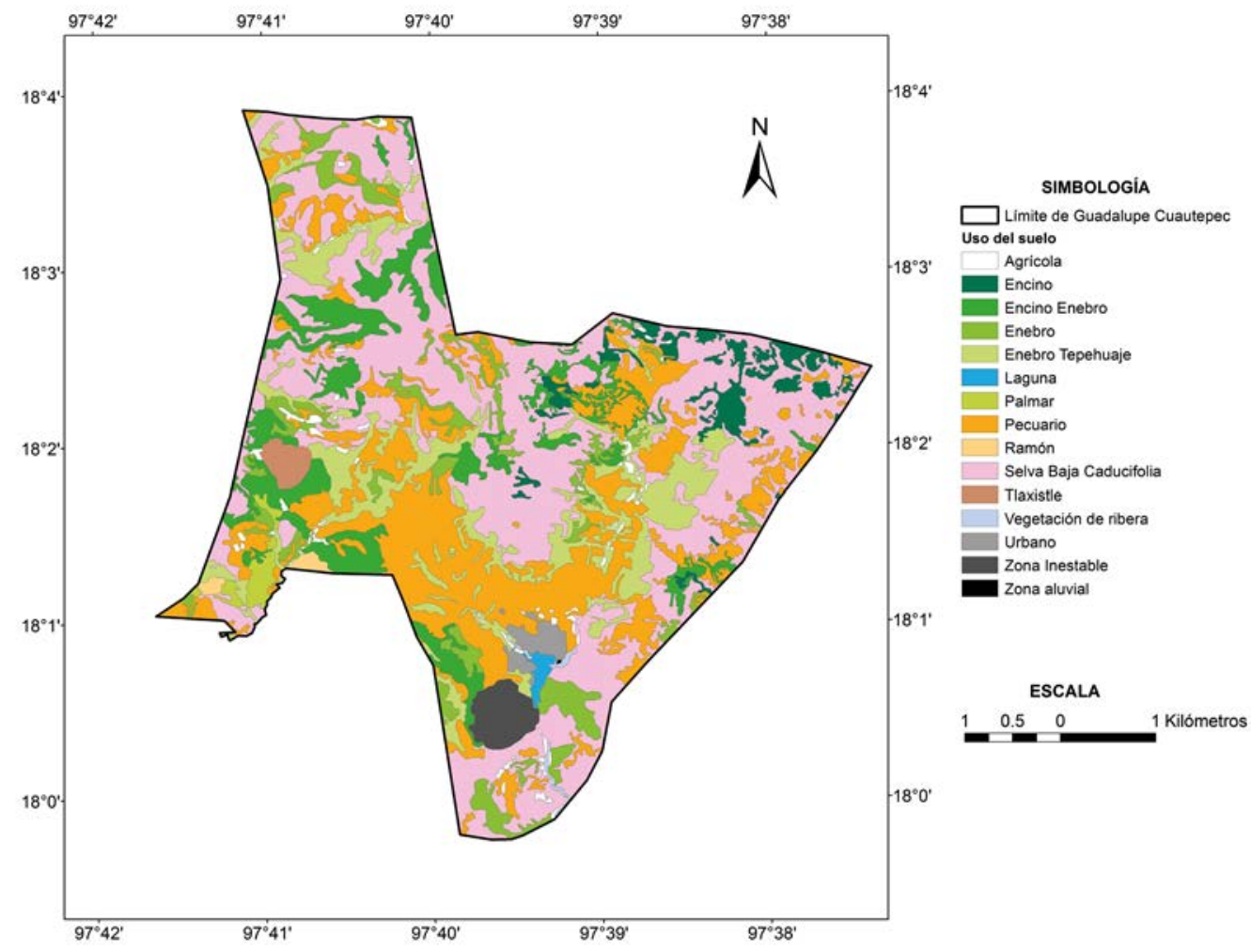

Figura 3. Tipos de uso de suelo. 


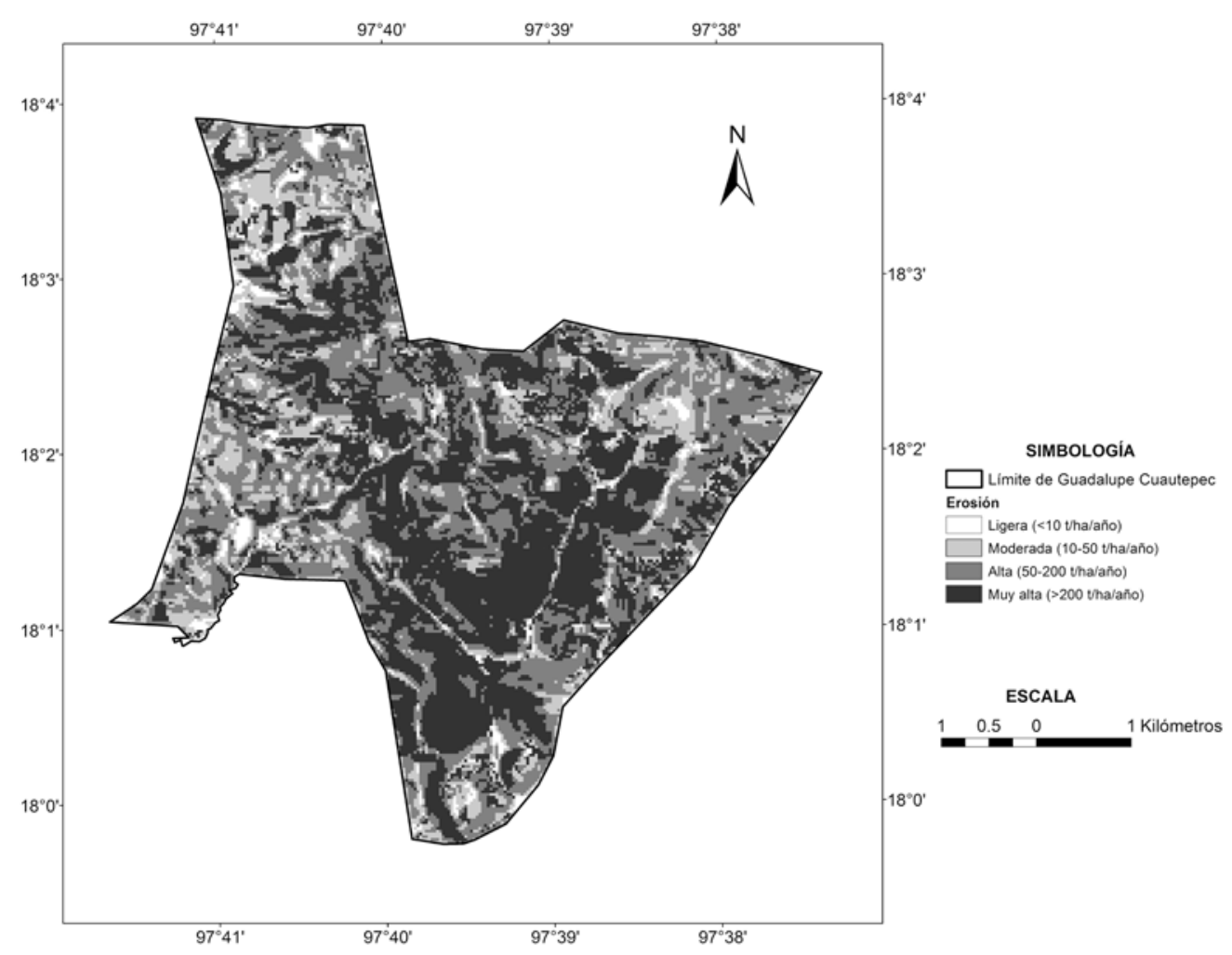

Figura 4. Nivel de erosión.

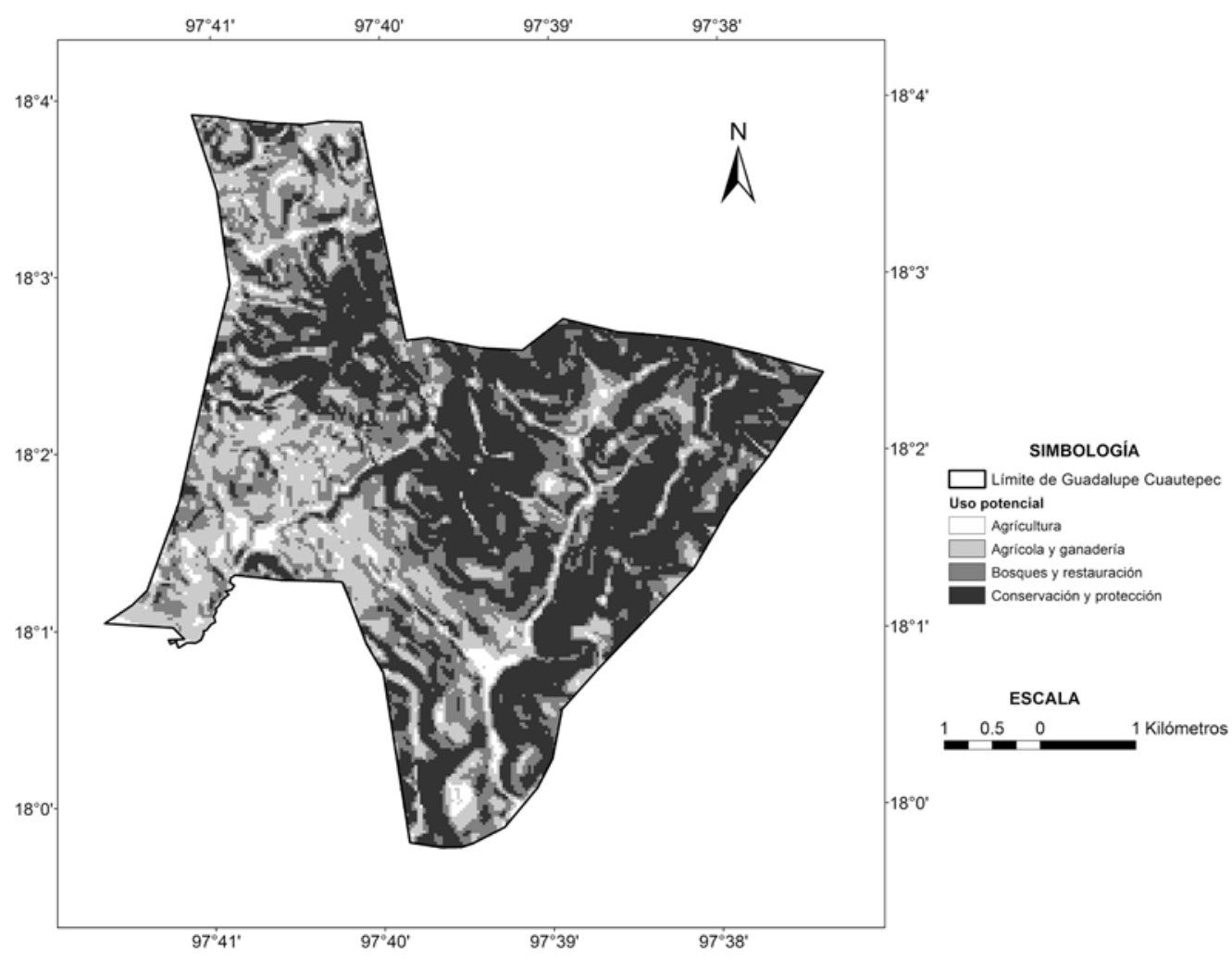

Figura 5. Uso potencial del suelo. 


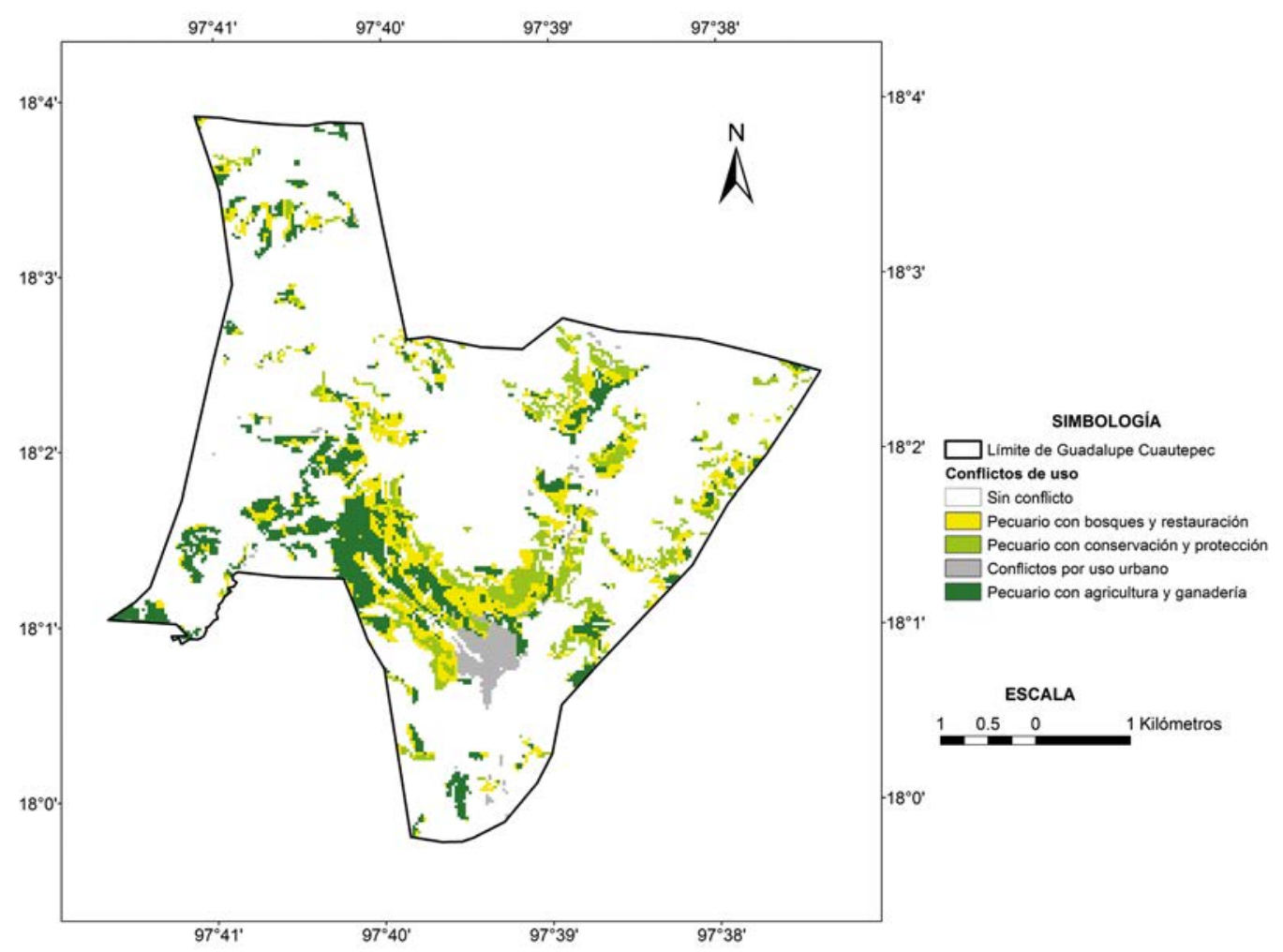

Figura 6. Conflictos del uso de suelo.

de vista comunitario los problemas asociados con el aprovechamiento y manejo de los recursos naturales. Se determinó que las principales causas que inciden directamente en la degradación de los recursos son: mala preparación del suelo agrícola, falta de obras de conservación de suelo, sobre pastoreo, tala inmoderada, falta de reforestación, caza de animales silvestres, mal uso del agua, basura en barrancas. Por otro lado, como efectos de los problemas anteriores se establecieron los siguientes: pérdida de fertilidad del suelo agrícola, erosión del suelo, falta de madera, escasez de leña, escasez de pastos para el ganado, mayores escurrimientos, escasez de agua, contaminación ambiental y derrumbes.

El diagnóstico participativo, aportó elementos importantes para la planificación y manejo integral del territorio comunitario en espacio y tiempo, entre los más relevantes está el plan de acción comunitario (Tabla 3), que permitió identificar las acciones que la comunidad realizará para el manejo sostenido de sus recursos natura- les, así como, si las actividades se realizarán individualmente (en familia) en casa o en sus parcelas, o si la actividad es de tipo comunitario en los terrenos de uso común. Los objetivos de este plan de acción considerados como de máxima prioridad son: 1. Realizar obras de conservación de suelos, 2. Controlar el aprovechamiento forestal, 3. Reforestar zonas sin vegetación, 4. Tener control de la caza de animales silvestres y 5 . Usar eficientemente el agua.

Con base en los objetivos 1, 2, y 3 y la información técnica generada se realizó el ordenamiento territorial (Fig. 7). Se considera el área inestable por deslizamientos de suelo como una de las partes a mantener sin cambios, debido a que no es posible llevar a cabo actividades agropecuarias o forestales. Se propone mantener como zonas de conservación, aquellos terrenos que actualmente tienen cobertura vegetal mayor de $75 \%$ y que abarcan 1666.7 ha, donde el nivel de deterioro es relativamente bajo y se mantienen mecanismos naturales de regeneración. Los terre- 
TABla 3. Plan de acción comunitario realizado por los habitantes de Guadalupe Cuautepec.

\begin{tabular}{|c|c|c|}
\hline Objetivo & Actividad & Recursos \\
\hline $\begin{array}{l}\text { Realizar obras de } \\
\text { conservación de } \\
\text { suelos. }\end{array}$ & $\begin{array}{l}\text { Construcción de bordos, presas de gaviones, pretiles en laderas } \\
\text { y tierras de cultivos, represas en arroyos y barrancas } \\
\text { Mantenimiento de las obras de conservación } \\
\text { Barreras vivas }\end{array}$ & $\begin{array}{l}\text { Capacitación } \\
\text { Herramientas en general } \\
\text { Material disponible en cada sitio } \\
\text { Plantas nativas }\end{array}$ \\
\hline $\begin{array}{l}\text { Controlar el } \\
\text { aprovechamiento } \\
\text { forestal. }\end{array}$ & $\begin{array}{l}\text { Reforestación para obtener leña, madera, adornos, medicinales } \\
\qquad y \text { comestibles. } \\
\qquad \begin{array}{l}\text { Dar una edad para corte o poda } \\
\text { Prohibir cortar árboles pequeños }\end{array}\end{array}$ & $\begin{array}{l}\text { Capacitación } \\
\text { Vivero para plantas nativas } \\
\text { Herramientas en general }\end{array}$ \\
\hline $\begin{array}{l}\text { Reforestar zonas } \\
\text { sin vegetación. }\end{array}$ & $\begin{array}{l}\text { Concientización de las necesidades de reforestación } \\
\qquad \text { Reforestación con plantas nativas } \\
\text { Exclusión de zonas al pastoreo por } 10 \text { años }\end{array}$ & $\begin{array}{l}\text { Capacitación } \\
\text { Vivero para plantas nativas } \\
\text { Herramientas en general }\end{array}$ \\
\hline $\begin{array}{l}\text { Tener control de la } \\
\text { caza de animales. }\end{array}$ & $\begin{array}{l}\text { Dejar que se reproduzcan animales en peligro de extinción } \\
\qquad \text { Control de cacería } \\
\text { Realización de un reglamento para la caza. }\end{array}$ & $\begin{array}{l}\text { Capacitación } \\
\text { Concientización } \\
\text { Aplicación del reglamento }\end{array}$ \\
\hline & Concientización para el buen uso del agua & \\
\hline $\begin{array}{l}\text { Usar } \\
\text { eficientemente } \\
\text { el agua. }\end{array}$ & $\begin{array}{c}\text { Reuso del agua } \\
\text { Uso de medidores para controlar el gasto de agua } \\
\text { Implementación de sistema de captación de agua de lluvia en } \\
\text { techos de vivienda } \\
\text { Construcción bebederos para el ganado y darles mantenimiento } \\
\text { Utilización de sistemas de riego por goteo en cultivos. }\end{array}$ & $\begin{array}{c}\text { Capacitación } \\
\text { Material de conducción } \\
\text { y almacenamiento } \\
\text { Material de construcción } \\
\text { Sistema de riego por goteo }\end{array}$ \\
\hline
\end{tabular}

nos más erosionados con necesidades de restauración ocupan 609.34 ha. La restauración se propone realizar mediante la reforestación con especies vegetales herbáceas y leñosas nativas, que tienen la potencialidad de crecer en zonas muy alteradas, y que permitan la recuperación de la fertilidad del suelo y el restablecimiento de la flora y fauna nativa que aún sobrevive en los sitios hasta hoy inalterados. Si bien la superficie que requiere reforestación de manera permanente y continua es de 439.5 ha (17.6\%), se recomienda llevar a cabo la reforestación en las etapas descritas en la figura 7; iniciando en el paraje Cristo Rey como primera etapa (28.8 ha), ya que estos son los terrenos más erosionados. La superficie a reforestar por año dependerá de la disponibilidad de recursos financieros y de la participación comunitaria. Conjuntamente con la reforestación deberán construirse obras de conservación de suelo y agua, tales como presas de piedra acomodada, presas de gaviones y zanjas bordo.

\section{DISCUSIÓN}

El ordenamiento ecológico es un instrumento de la política ambiental diseñado para caracterizar, diagnosticar y proponer formas de utilización del territorio y de sus recursos naturales, bajo el enfoque de uso racional y diversificado con el acuerdo de la población (Negrete y Bocco, 2003). Con la participación comunitaria se identifican los problemas que la comunidad considera importantes haciendo partícipe a la comunidad no sólo del 


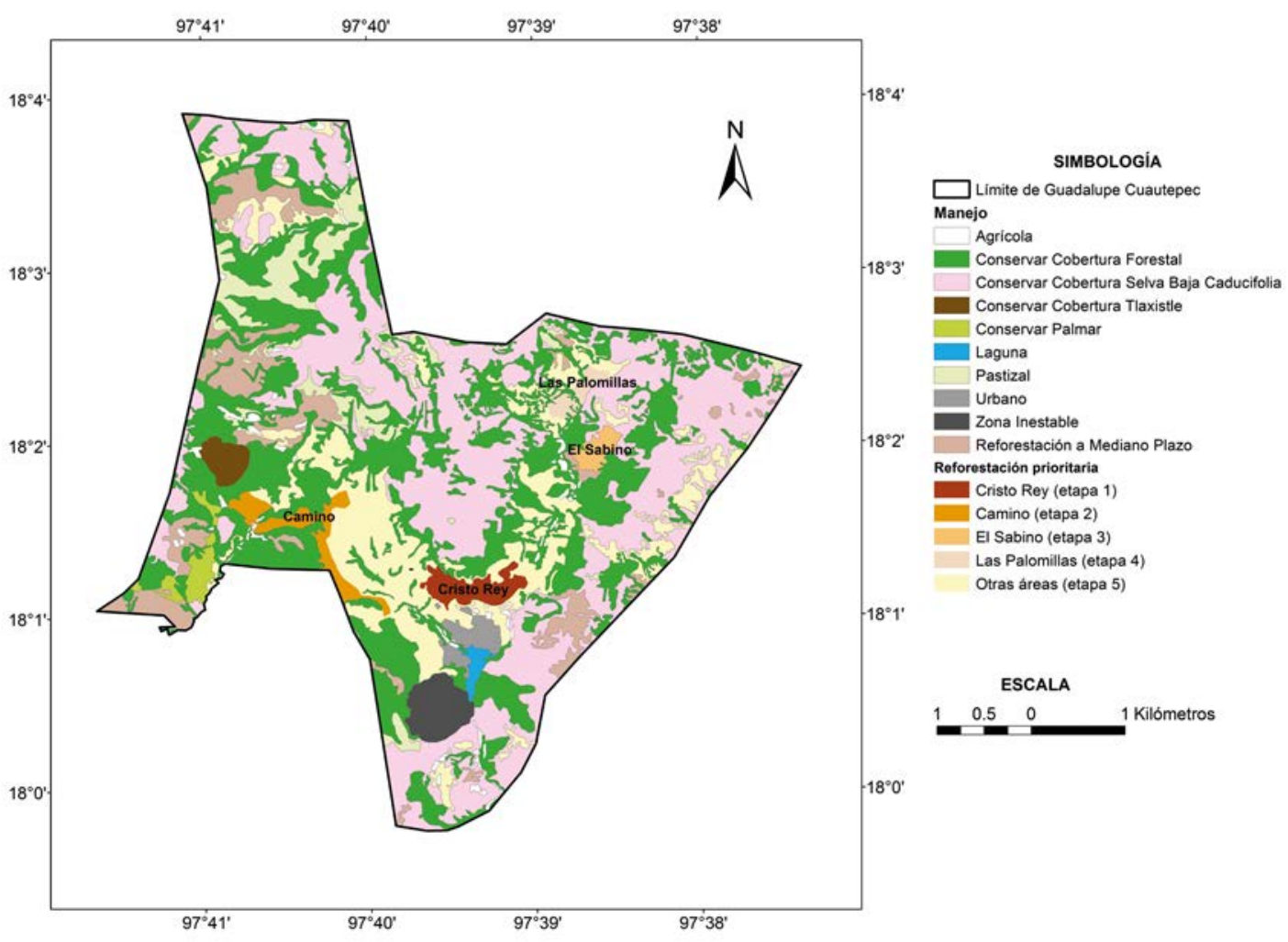

Figura 7. Ordenamiento ecológico territorial de Guadalupe Cuatepec.

diagnóstico, sino también del seguimiento y evaluación del proceso participativo (Expósito, 2003; Osorio y Contreras, 2009); y como bien mencionan Hernández et al. (2014) es ética y metodológicamente imprescindible el respeto y la asimilación de las culturas y conocimientos de dichas comunidades en los programas de ordenamiento, particularmente los comunitarios, como el de este estudio.

Entre los problemas debidos a la degradación de los recursos naturales identificados en el diagnóstico participativo realizado, están la pérdida de fertilidad del suelo agrícola, la falta de madera y leña y la escasez de pasto para el ganado. Tales problemas están asociados con el uso intensivo de los recursos naturales y con la realización de actividades agropecuarias en zonas no aptas para tales usos; esta misma situación se ha observado en diferentes estudios enfocados al manejo de los recursos naturales en comunidades rurales (GAIA, 2004; Grupo Mesófilo, 2011; LagunasVázquez et al., 2008; Meraz et al., 2011; Negrete y Aguilar, 2006; Pujadas, 2003; Robles-Guadarrama, et al, 2005).
El ordenamiento ecológico territorial propuesto considera mantener zonas de conservación y realizar la restauración con especies nativas, con el consentimiento de la comunidad mediante su validación en asambleas, lo cual es similar a lo propuesto en el ordenamiento territorial comunitario por GAIA (2004), en San Felipe Lachilló, Xanica, Oaxaca. Bocco et al. (2000) consideran que las comunidades rurales dependen en gran medida de sus recursos naturales, lo que demuestra su importancia para resguardarlos, ya que ellas pueden operar como aliadas de la protección biológica y la diversidad genética in situ. En este mismo contexto Almazán-Núñez et al., (2011) mencionan que el ordenamiento territorial permite establecer áreas de conservación comunitaria, las cuales son una alternativa viable para la conservación, la biodiversidad y los servicios ambientales.

Es necesario crear un comité de recursos naturales representado por líderes naturales de la comunidad que permita el seguimiento y evaluación en las actividades 
para el manejo de los recursos naturales, debido a que las autoridades municipales o comunales se cambian en periodos cortos, como se propone también en el caso del ordenamiento territorial de la comunidad de San Juan Chicomezuchil, Ixtlán, Oaxaca (Grupo Mesófilo, 2011).

\section{CONCLUSIONES}

La participación de los integrantes de la comunidad permitió identificar el tipo de acciones que la comunidad realizará para el manejo sustentable de sus recursos naturales mediante un plan de acción comunitario.

En el ordenamiento territorial se consideraron tanto los objetivos establecidos en el plan de acción comunitario, así como la información técnica generada. Se propone mantener como zonas de conservación 1666.7 ha que tienen cobertura vegetal mayor de $75 \%$, que incluye áreas que actualmente soportan vegetación de encino, selva baja caducifolia, enebro, tepehuaje, tlaxistle y palma de sombrero. Los terrenos más erosionados con necesidades de restauración ocupan $24.43 \%$ (609.3 ha).

\section{RECONOCIMIENTOS}

Los resultados de este documento forman parte del proyecto financiado por la Comisión Nacional de Áreas Naturales Protegidas (Conanp) con número de convenio Conanp/DR08/RP07/20/Procodes/16/10.

\section{REFERENCIAS}

Almazán-Núñez, R. C., A. Almazán-Juárez, F. Ruiz-Gutiérrez. 2011. Áreas comunitarias para la conservación de los recursos biológicos de la Sierra Madre del sur, Guerrero, México. Universidad y Ciencia 27(3):315-329.

Bautista-Sánchez, G., E.C. Pedro-Santos y G. Álvarez-Olguín. 2013. Participación y acción comunitaria en el manejo de recursos naturales de uso común en la Mixteca Oaxaqueña. Ra Ximhai 9(2):89-98.

Blanco A., A., S. Martínez R., O. Sánchez P., A. Rubio S., C. Cisneros C., E.C. Pedro S., R. Morales L. y F. Sustaita R. 2001. Aplicación de un modelo de balances hídricos en la cuenca alta del río Mixteco (Oaxaca). Determinación del binomio infiltración/escurrimiento con vistas a la recons- trucción de sus ecosistemas forestales. Universidad Tecnológica de la Mixteca, Huajuapan de León, Oaxaca. México. 250 p.

Bocco, G., A. Velázquez y A. Torres. 2000. Ciencia, comunidades indígenas y manejo de recursos naturales. Un caso de investigación participativa en México. Interciencia 25(2):64-70.

Conabio (Comisión Nacional para el Conocimiento y Uso de la Biodiversidad). 2009. Capital natural de México. Síntesis. Conabio. México. 104 p.

Cotler, H. 2004. El manejo integral de cuencas en México: estudios y reflexiones para orientar la política ambiental. $2^{\mathrm{a}}$ ed. Instituto Nacional de Ecología. México. 345 p.

Expósito, V.M. 2003. Diagnóstico rural participativo. Una guía práctica. Centro Cultural Poveda. Santo Domingo, República Dominicana. 118 p.

GAIA (Grupo Autónomo para la Investigación Ambiental). 2004. Estudio de ordenamiento territorial comunitario de San Felipe Lachilló, Xanica, Miahuatlán, Oaxaca. 104 p.

García-Mendoza, A.J., M.J. Ordóñez y M. Briones-Salas, eds. 2004. Biodiversidad de Oaxaca. Universidad Nacional Autónoma de México. Fondo Oaxaqueño para la Conservación de la Naturaleza. World Wildlife Fund. México. 605 p.

Grupo Mesófilo. 2011. Estudio de Ordenamiento Territorial Comunitario en San Juan Chicomezuchil, Ixtlán, Oaxaca. Grupo Mesófilo A.C. Oaxaca, México. 151 p.

Hernández S., J.R., M. Bollo M., A.P. Méndez L. y L.M. Espinosa-Rodríguez. 2014. Panorama contemporáneo del ordenamiento ecológico territorial en México. Polígonos. Revista de Geografía 26:111-146.

Inegi (Instituto Nacional de Estadística Geografía e Informática). 2003. Continuo de elevaciones mexicano (CEM) escala 1:50 000 [en línea]. [Consulta 5 de diciembre de 2010]. Disponible en: http://mapserver.inegi.org.mx/ DescargaMDEWeb/?s=geo\&c=977.

Inegi (Instituto Nacional de Estadística y Geografía). 2010. Censo de población y vivienda 2010 [en línea]. [Consulta 07 de enero de 2016]. Disponible en: http://www.inegi. org. $\mathrm{mx} / \mathrm{sistemas} / \mathrm{consulta}$ _resultados/iter 2010 . aspx?c=27329\& $2=$ est.t 
Klingebiel, A.A. y P.H. Montgomery. 1961. Land capability classification (No. 210). Soil Conservation Service, US Department of Agriculture. Washington, DC, EUA. 21 p.

Lagunas-Vázquez, M., L.F. Beltrán-Morales, J. Urciaga-García y A. Ortega-Rubio. 2008. Evaluación rural participativa: uso de los recursos naturales en la reserva de la biosfera El Vizcaíno, BCS, México. Economía, sociedad y territorio $8(26): 451-476$.

Meraz J., A.D.J., J.L. Galarza M., J. Sosa R., A. Ponce M. y J.A. Torres G. 2011. Ordenamiento ecológico comunitario: un modelo de manejo de recursos naturales para el desarrollo comunitario. Estudio de caso ejido Potrero de los López, Aguascalientes. Revista Chapingo. Serie ciencias forestales y del ambiente 17(1):151-163.

Negrete F., G. y E. Aguilar U. 2006. Territorios rurales, política de planeación y ordenamiento ecológico local-comunitario en México. In: S. Anta F., A. Arreola M., M. González O. y J. Acosta G, eds. Ordenamiento territorial comunitario. Instituto Nacional de Ecología (INE-Semarnat). México, D.F. p:19-40.

Negrete, G. y G. Bocco. 2003. El ordenamiento ecológico comunitario: una alternativa de planeación participativa en el contexto de la política ambiental de México. Gaceta ecológica (68):9-22.
FAO (Organización de la Naciones Unidas para la Alimentación y la Agricultura). 1980. Metodología provisional para la evaluación de la degradación de los suelos. Roma. 86 p.

Osorio R., M.L. y A. Contreras. 2009. El diagnóstico rural participativo y el manejo de los recursos naturales. Estudios Agrarios, Secretaría de la Reforma Agraria (42):109-136. Pujadas B., A. 2003. Comunicación y participación social en el programa de ordenamiento ecológico territorial de la costa de Jalisco y la Reserva de la Biosfera Chama-Cuixmala. Tesis de maestría. Universidad Nacional Autónoma de México, Instituto de Ecología. México. 291p.

Robles-Guadarrama., C., A. Flores, C. Cabrera M., C. Santos y G. Vidriales C. 2005. Ordenamiento comunitario participativo del ejido Encino Amarillo municipio de Mecayapan, Veracruz. 62p.

Manuscrito recibido el 20 de febrero de 2015. Aceptado el 9 de febrero de 2016.

Este documento se debe citar como:

Álvarez-Olguín, G., F. Sustaita-Rivera, G. Bautista-Sánchez y E.C. Pedro-Santos. 2016. Ordenamiento ecológico territorial de Guadalupe Cuautepec, San Juan Bautista Suchitepec, Oaxaca, desde una perspectiva técnica y comunitaria. Madera y Bosques 22(1):155-167. 